Regular Paper

\title{
Leakage Flux Recovery Coil for Energy Harvesting Using Magnetoplated Wire
}

\author{
Tatsuya YAMAMOTO*1, Yinggang BU*1 and Tsutomu MIZUNO*1
}

\begin{abstract}
Magnetic flux leakage from an AC adapter or a notebook PC can be recovered by using a coil. However, because the magnetic flux that leaks out is very small, the power that can be collected by a coil is small. As a result, the quality factor of the coil must be increased to increase the power that can be collected by the coil. The use of magnetoplated wire (MPW) to from the coil can reduce the resistance due to the proximity effect in comparison with the use of copper wire (COW). MPW is a COW that has a circumference that is plated with a magnetic thin film. In this paper, we investigate the energy harvesting by a coil consisting of MPW. We used a coil with an outer diameter of $9.9 \mathrm{~mm}$ and 300 turns. The quality factor of the leakage flux recovery coil using COW and MPW at a frequency of $f=250$ $\mathrm{kHz}$ was 119 and 135 , respectively, and it is increase in $13.4 \%$ by using MPW.
\end{abstract}

Keywords: energy harvesting, leakage flux, magnetoplated wire, LC series resonance, AC resistance, quality factor. (Received: 24 July 2014, Revised: 27 April 2015)

\section{Introduction}

There is an abundance of low-level energy in the environmental, such as light and heat, vibrations, and electromagnetic waves that can be converted to electricity via energy harvesting technology. Recently, such energy harvesting technology has attracted increasing attention [1-3].

One form of energy in the environment is leakage magnetic flux. The magnetic flux is leaked from personal computers and electronics products and can be converted to electric power by using a coil. Energy harvesting from magnetic flux at the commercial frequencies has already been examined [2]. In addition, the energy harvesting from electromagnetic waves of frequencies greater than several hundred $\mathrm{MHz}$ has been examined [3]. However, there are few considered examples regarding the magnetic flux at other frequencies. Therefore, we focused on using a coil to perform energy harvesting from magnetic flux at a frequency of several hundred $\mathrm{kHz}$ that leaks out from a PC or an $\mathrm{AC}$ adapter.

The electrical power collected from leakage magnetic flux is proportional to the quality factor of the leakage flux recovery coil [4]. However, the AC resistance increases due to the skin effect and a proximity effect when the magnetic flux crosses a coil. Higher AC resistances decrease the quality factor of the coil, thereby decreasing the electric power collected from leakage magnetic flux. As a result, the quality factor of the coil must be increased to collect more electric power from the leakage magnetic flux.

\footnotetext{
Correspondence: T. YAMAMOTO, Faculty of Engineering, Shinshu University, 4-7-1 Wakasato, Nagano 380-8553, Japan

email: 14tm243b@shinshu-u.ac.jp

${ }^{*}$ Shinshu University
}

To reduce the $\mathrm{AC}$ resistance and increase the quality factor of the coil, we proposed using magnetoplated wire (MPW) as the winding wire of the leakage flux recovery coil [5]. MPW has a structure of a magnetic thin film that is plated onto the circumference of a copper wire (COW). By using MPW for the coil, because an AC magnetic field passes the magnetic film of magnetic permeability and has a specific resistance greater than that of copper, the eddy current loss occurring in MPW is reduced in comparison with COW. In addition, the inductance of the coil increases because the magnetic film has high magnetic permeability. Thus, the quality factor of the coil is higher using MPW versus COW.

In this paper, the impedance characteristics of the leakage flux recovery coil for energy harvesting using COW and MPW are compared. In addition, a compareson of the output characteristics of the energy harvesting circuit using coils of COW and MPW when a uniform magnetic flux is interlinked to the coil is performed. We also investigated the energy harvesting from a generalpurpose notebook PC. We discussed the following matters:

1) Impedance characteristics of the leakage flux recovery coil.

2) Output characteristics of the energy harvesting circuit.

3) Energy harvesting from a notebook PC.

\section{Structure and operation principal of the energy harvesting circuit}

\subsection{Structure of the Winding Wires}

Figure 1 shows the structure of the winding wire using a leakage flux recovery coil. The COW has a diameter of $90 \mu \mathrm{m}$ and is plated with an insulating film of $8 \mu \mathrm{m}$ thickness. The MPW is a COW with a diameter of $90 \mu \mathrm{m}$ and is plated with magnetic thin films ( $\mathrm{Fe}$ and 


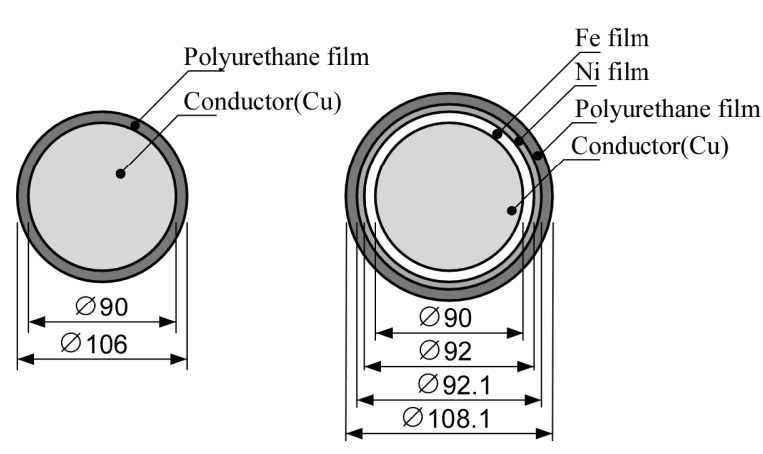

(a) $\mathrm{COW}$

(b) MPW

Fig. 1. Structure of the winding wire for the coil (units: $\mu \mathrm{m}$ ).

Ni). The thickness of the Fe and Ni thin films are $1 \mu \mathrm{m}$ and $0.05 \mu \mathrm{m}$, respectively. The Ni film is plated for the ease of soldering.

\subsection{Structure of the Leakage Flux Recovery Coils}

Figure 2 shows the structure of a leakage flux recovery coil. The outer diameter, inside diameter and axial length of the coil are $9.9 \mathrm{~mm}, 4.5 \mathrm{~mm}$ and $2.2 \mathrm{~mm}$ respectively. The winding wire of the coil is COW and MPW, as shown in Fig. 1. There are 300 turns in the coil. The core material of the coil is ferrite (FE20).

\subsection{Operating Principal of the Energy Harvesting Circuit}

Figure 3 shows the structure of the energy harvesting circuit. The magnetic flux density that crosses the leakage flux recovery coil is $\mu_{0} H(\mathrm{~T})$. The capacitor for the resonance is connected to the coil in series. The coil and capacitor resonance is at the frequency of the magnetic flux that crosses the coil.

Figure 4 shows the equivalent circuit of the energy harvesting circuit. $V$ is the induced voltage when the magnetic flux that crosses the coil. $L$ and $R$ is the inductance and resistance of the coil, respectively $R_{\mathrm{L}}$ is the output resistance and that is connected to the coil and to capacitor $C$.

From Faraday's law, the induced voltage $V$ of the leakage flux recovery coil is given by (1) [4].

$$
V=\frac{\mathrm{d} \Phi}{\mathrm{d} t}=N_{C} \frac{2 \pi^{2} r_{\mathrm{a}}{ }^{2} f \mu_{0} \mu_{\mathrm{r}} H}{N}(\mathrm{~V})
$$

where $\Phi$ is the magnetic flux crossing to the coil (Wb), $N_{\mathrm{c}}$ is the number of turns of the coil, $r_{\mathrm{a}}$ is the average radius of the coil $(\mathrm{m}), f$ is the frequency of magnetic flux density crossing to the coil $(\mathrm{Hz}), \mu_{0}$ is the permeability of ferrite core $(\mathrm{H} / \mathrm{m}), \mu_{\mathrm{r}}$ is the permeability of ferrite core, and $N$ is the demagnetizing factor.

The induced voltage is proportional to the number of turns of the coil, the radius, and the frequency of the magnetic flux crossing the coil.

When the coil and capacitor in the energy harvesting are in circuit resonance, from the law of the maximum

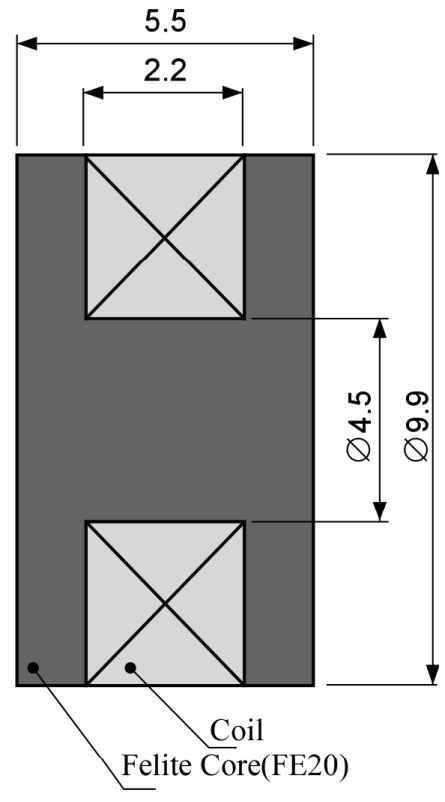

Fig. 2. Structure of the leakage flux recovery coil (units: $\mathrm{mm}$ ).

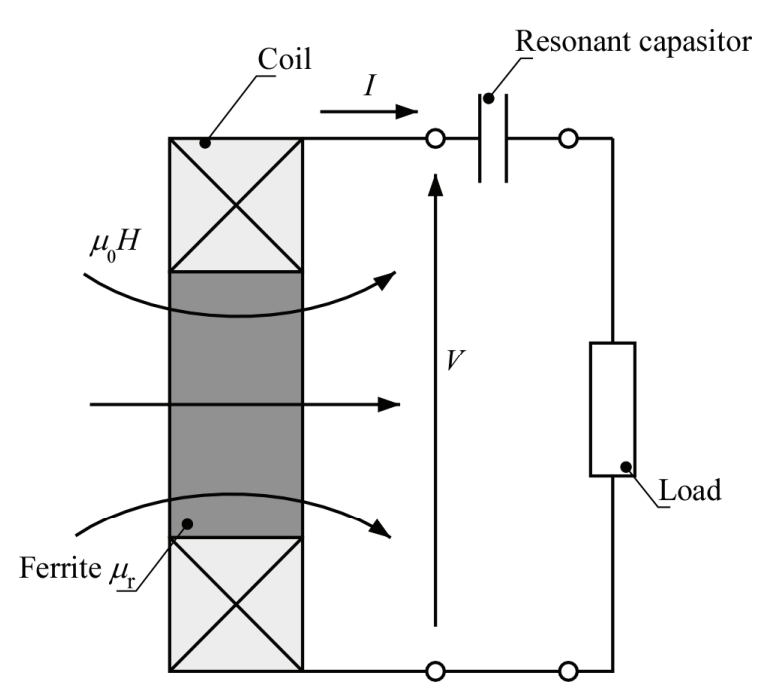

Fig. 3. Operating principle of the energy harvesting circuit.

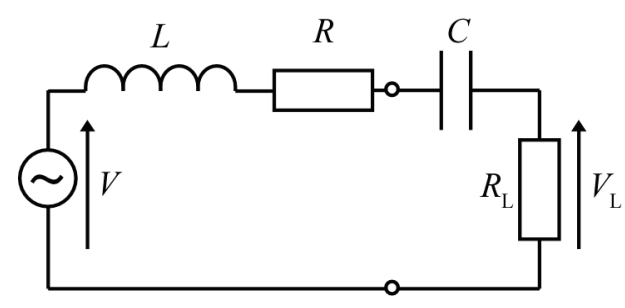

Fig. 4. Equivalent circuit of energy harvesting circuit. 
power supply, the output power $P$ of an energy harvesting circuit is given by (2).

$$
P=\frac{V_{\mathrm{L}}^{2}}{R_{\mathrm{L}}}=\frac{V^{2}}{4 R}(\mathrm{~W})
$$

where $V_{\mathrm{L}}$ is the output voltage of the energy harvesting circuit (V).

Output power $P$ becomes (3) in consideration of the resistance $R$, inductance $L$ and quality factor $Q$ of the coil.

$$
P=Q f \times \frac{\pi^{3} r_{\mathrm{a}}^{2} l_{\mathrm{a}} \mu_{\mathrm{r}}{ }^{2}}{2 K N^{2}} \times\left(\mu_{0} H\right)^{2}(\mathrm{~W})
$$

where $K$ is the coefficient involving the inductance.

The output power of the energy harvesting circuit is proportional to the quality factor of the coil when the magnetic flux density crossing the coil is uniform.

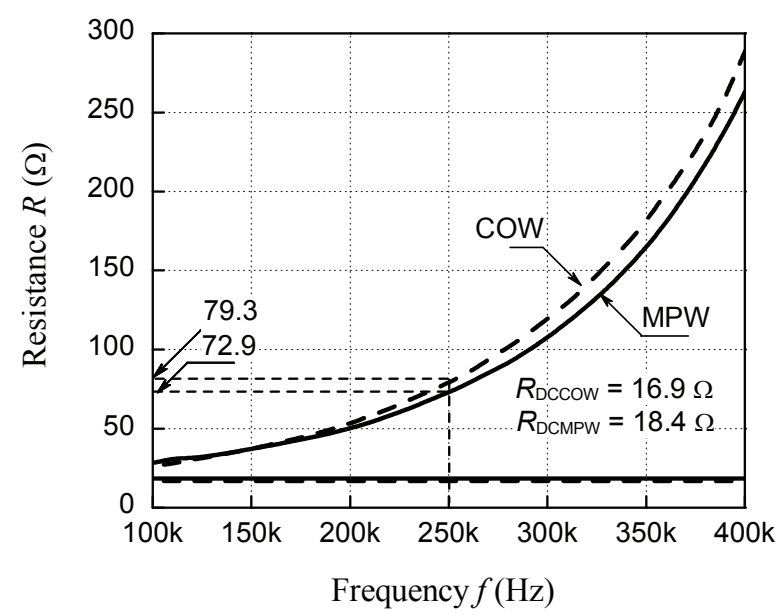

(a) Resistance

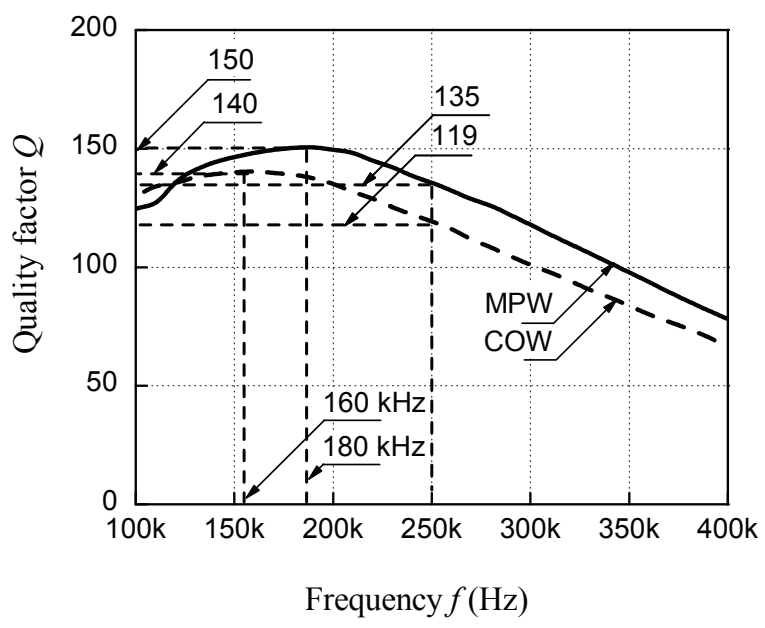

(c) Quality factor

\section{Impedance and Output Characteristics of the Leakage Flux Recovery Coil}

\subsection{Impedance Characteristics of the Coils}

Figure 5 shows the impedance vs. frequency characteristics of the leakage flux recovery coils using COW and MPW. The impedance was measured using an impedance analyzer (Agilent Technologies, 4294A).

Figure 5(a) shows the resistance vs. frequency characteristic of the coil. The self-resonant frequency $f_{0}$ of the leakage flux recovery coil using COW and MPW was $730 \mathrm{kHz}$ and $700 \mathrm{kHz}$, respectively. The resistance $\mathrm{R}$ of the leakage flux recovery coil using COW and MPW at frequency $f=250 \mathrm{kHz}$ was $79.3 \Omega$ and $72.9 \Omega$, respectively; thus, the resistance of MPW decreased by $8.8 \%$ compared with that of COW. This finding is due to the restraint of the proximity effect of the coil using

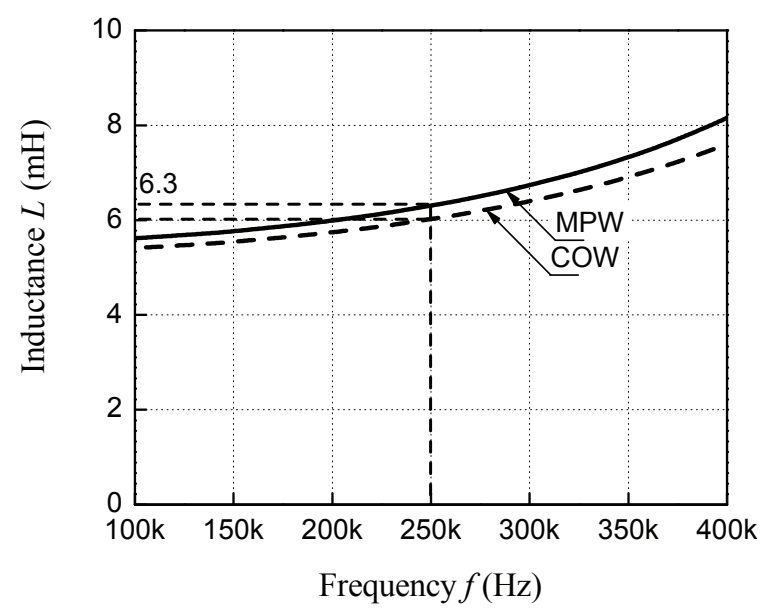

(b) Inductance

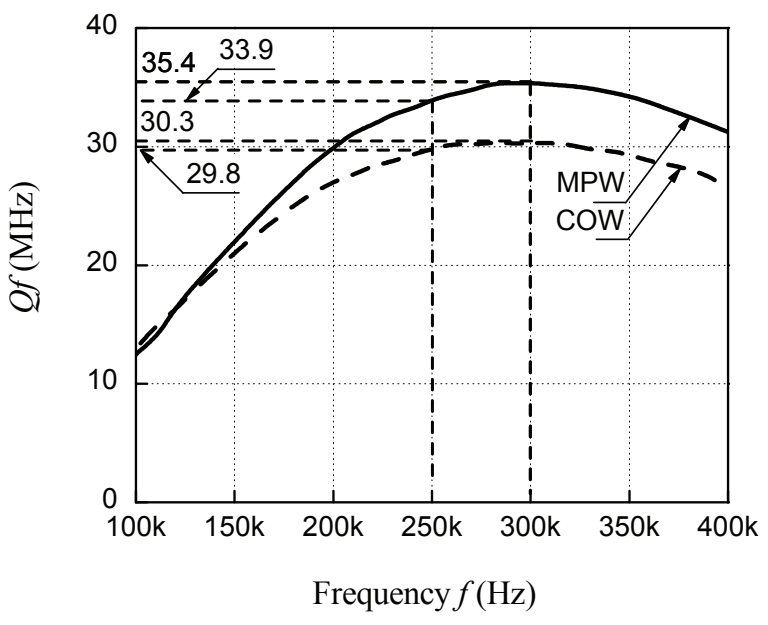

(d) $Q f$

Fig. 5. Impedance vs. frequency characteristics of the leakage flux recovery coil $\left(f_{0 \mathrm{cow}}=730 \mathrm{kHz}, f_{0 \mathrm{MPW}}=700 \mathrm{kHz}\right)$. 
MPW.

Figure 5(b) shows the inductance $L$ vs. frequency characteristics of the coil. The inductance $L$ of the leakage flux recovery coil using COW and MPW at frequency $f=250 \mathrm{kHz}$ was $6 \mathrm{mH}$ and $6.3 \mathrm{mH}$, respectively; thus, the inductance of MPW increased by $5 \%$ compared with that of COW. This finding is due to store the magnetic energy in a magnetic thin film of the coil using MPW.

Figure 5(c) shows the quality factor $Q$ vs. frequency characteristics of the coil. The quality factor $Q$ of the leakage flux recovery coil using COW and MPW at frequency $f=250 \mathrm{kHz}$ was 119 and 135 , respectively; thus, the inductance of MPW increased by $13.4 \%$ compared with that of COW. This finding is due to the reduction of the $\mathrm{AC}$ resistance and the increase of the inductance of the coil using MPW.

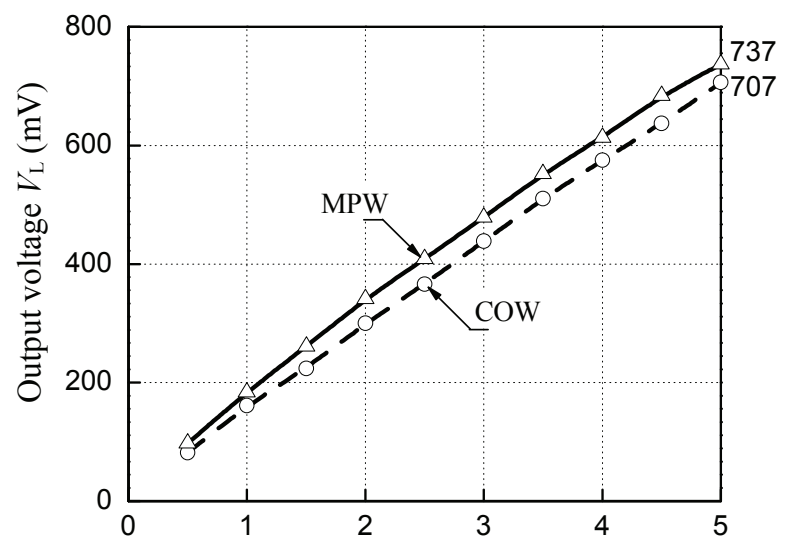

Flux density $B(\mu \mathrm{T})$

(a) Output voltage
Figure 5(d) shows the $Q f$ vs. frequency characteristics calculated from Fig. 5(c). The $Q f$ of the leakage flux recovery coil using COW and MPW at frequency $f=$ $250 \mathrm{kHz}$ was $30.3 \mathrm{MHz}$ and $33.9 \mathrm{MHz}$, respectively; thus, $Q f$ of MPW increased by $11.9 \%$ compared with that of COW.

\subsection{Output Characteristics of the Energy Harvesting Circuit}

Figure 6 shows the output voltage and power vs. magnetic flux density characteristics of the energy harvesting circuit in a uniform magnetic flux at frequency $f=250 \mathrm{kHz}$ [6]. We measured the output voltage and power when magnetic flux density $B$ is changed in the range of 0.5 to $5 \mu \mathrm{T}$ at the frequency $f=250 \mathrm{kHz}$. We set the magnetic flux density to $5 \mu \mathrm{T}$ because the mag-

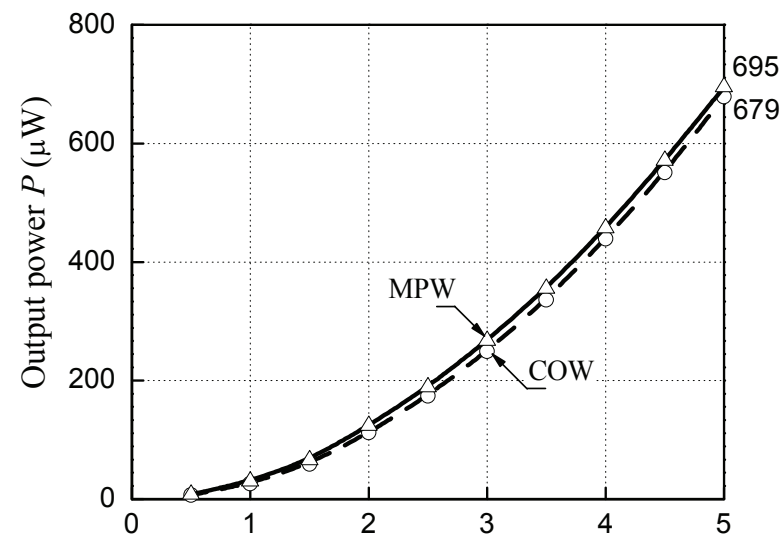

Flux density $B(\mu \mathrm{T})$

(b) Output power

Fig. 6. Output voltage and power vs. flux density characteristics of the energy harvesting circuit $(f=250 \mathrm{kHz})$.

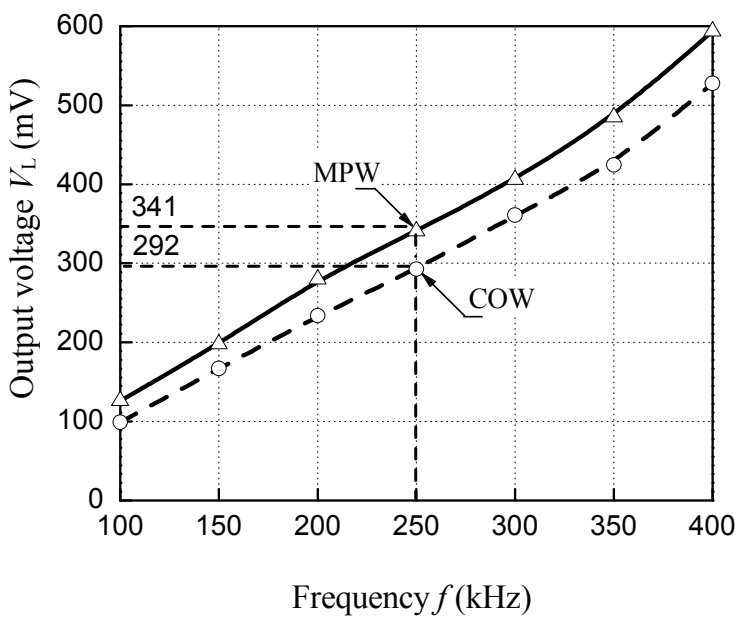

(a) Output voltage

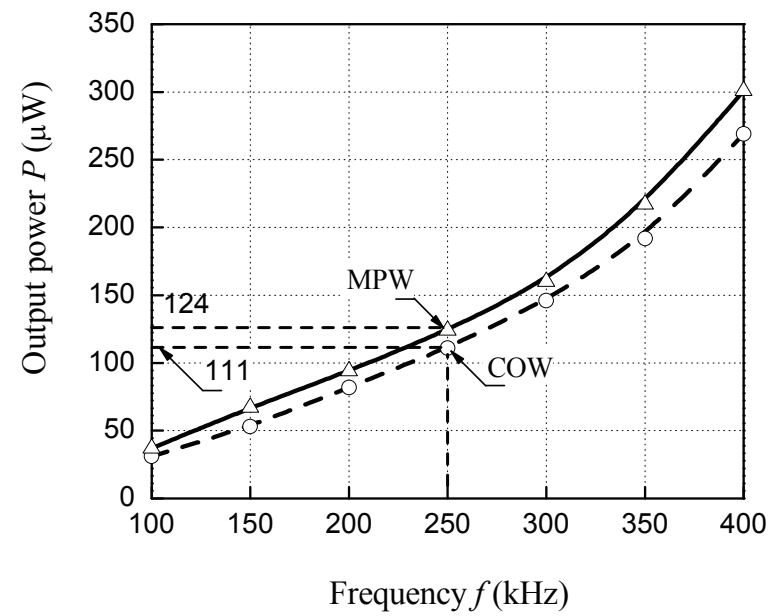

(b) Output power

Fig. 7. Output voltage and power vs. frequency characteristics of the energy harvesting circuit $(B=2 \mu \mathrm{T})$. 
netic flux density that leaks from the notebook PC is very small.

Figure 6(a) shows the output voltage $V_{\mathrm{L}}$ vs. magnetic flux density $B$ characteristics of the energy harvesting circuit. The output voltage of the energy harvesting circuit using COW and MPW at frequency $f=250 \mathrm{kHz}$ and flux density $B=5 \mu \mathrm{T}$ was $707 \mathrm{mV}$ and $737 \mathrm{mV}$, respectively; thus, the output voltage of MPW increased by $4.2 \%$ compared with that of COW.

Figure 6(b) shows the output power $P$ vs. magnetic flux density $B$ characteristics of the energy harvesting circuit. The output power of the energy harvesting circuit using COW and MPW at frequency $f=250 \mathrm{kHz}$ and flux density $B=5 \mu \mathrm{T}$ was $679 \mu \mathrm{W}$ and $695 \mu \mathrm{W}$, respectively; thus, the output voltage of MPW increased by $2.3 \%$ compared with that of COW. This finding is due to the increase of the quality factor $Q$ of the coil using MPW.

Figure 7 shows the output voltage and power vs. frequency characteristics of the energy harvesting circuit in uniformed magnetic flux at the magnetic flux density $B$ $=2 \mu \mathrm{T}$. We measured the output voltage and power when the frequency $f$ was changed over the range of 100 to $400 \mathrm{kHz}$ at the magnetic flux density $B=2 \mu \mathrm{T}$.

Figure 7(a) shows the output voltage $V_{\mathrm{L}}$ vs. frequency $f$ characteristics of the energy harvesting circuit. The output voltage of the energy harvesting circuit using COW and MPW at frequency $f=250 \mathrm{kHz}$ and flux density $B=2 \mu \mathrm{T}$ was $292 \mathrm{mV}$ and $341 \mathrm{mV}$, respectively; thus, the output voltage of MPW increased by $16.8 \%$ compared with that of COW.

Figure 7 (b) shows the output power $P$ vs. frequency $f$ characteristics of the energy harvesting circuit. The output power of the energy harvesting circuit using COW and MPW at frequency $f=250 \mathrm{kHz}$ and flux density $B=2 \mu \mathrm{T}$ was $111 \mu \mathrm{W}$ and $124 \mu \mathrm{W}$, respectively; thus, the output voltage of MPW increased by $11.7 \%$ compared with that of COW. This finding is due to the increase of the quality factor $Q$ of the coil using MPW.

\section{Energy Harvesting from Notebook PC}

\subsection{Structure of Energy Harvesting Circuit}

Figure 8 shows the circuit of the energy harvesting from a notebook PC. The energy harvesting circuit was composed of a leakage flux recovery coil, a resonant capacitor, a four times voltage rectifier circuit, a charging capacitor and a wireless module (PTM330 Enocean). The capacitor is connected to the coil in series for resonance. The coil and capacitor resonate at the frequency of the magnetic flux that leaks from notebook PC. A rectifying circuit is a four times voltage rectifying circuit, and the charging capacitor $C_{\mathrm{o}}$ is $100 \mu \mathrm{F}$. We investigated the operation of the wireless module to connect the charging capacitor that was charged by the energy harvesting circuit.

Figure 9 shows the operating range of the wireless module. It requires a charging voltage $V_{\mathrm{o}}=3 \mathrm{~V}$ and a

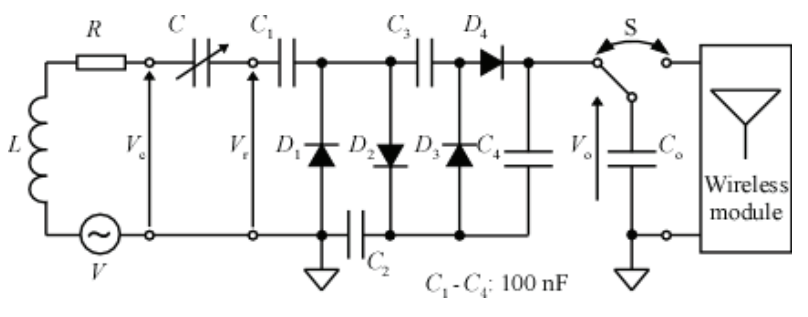

Fig. 8. Structure of the energy harvesting circuit.

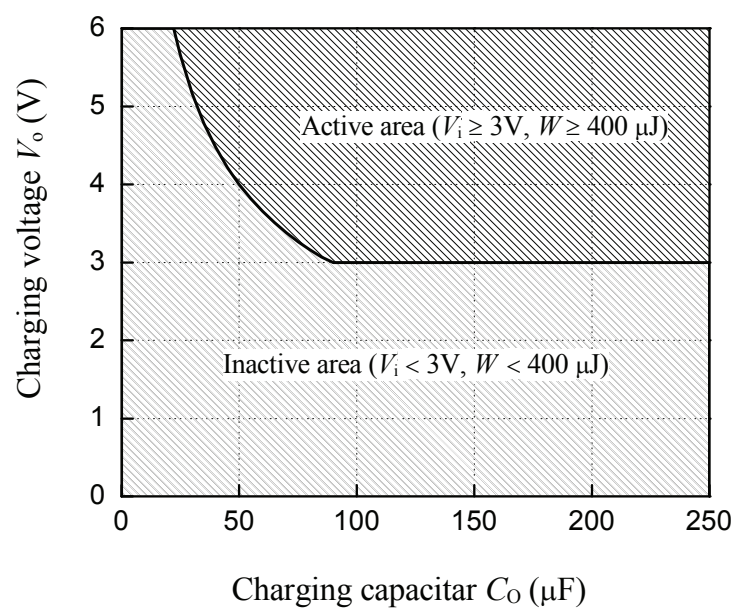

Fig. 9. Operating range of the wireless module.

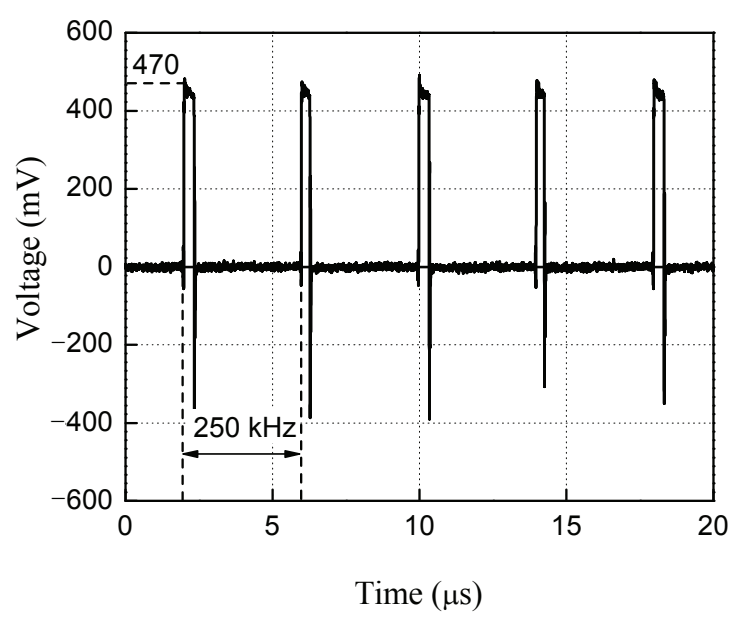

Fig. 10. Search voltage waveform of a notebook PC.

charging energy $W=400 \mu \mathrm{J}$ to capacitor $C_{\mathrm{o}}$ to operate the wireless module.

\subsection{Energy Harvesting from a Notebook PC}

We investigated energy harvesting from a household electrical appliance. The object of the energy harvesting 


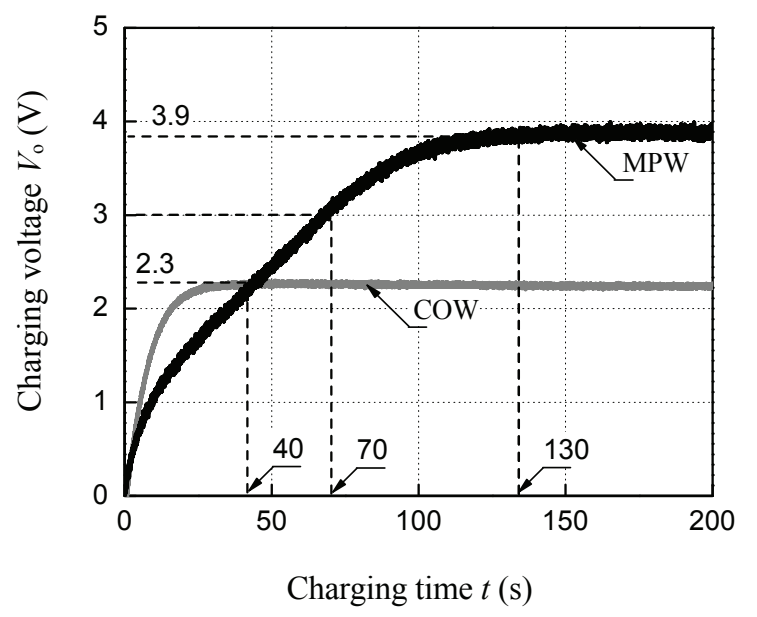

Fig. 11. Charging characteristics of the energy harvesting circuit.

is a general-purpose notebook PC (SONY VGN-G2). The leakage flux recovery coil is installed in the upper part of the motherboard where an inductor was implemented.

Figure 10 shows the voltage waveform using a search coil for detection at the measurement point of the notebook PC. The magnetic flux that leaks from the notebook PC was in the form of a pulse at frequency of $250 \mathrm{kHz}$. From Fig. 10, we set the resonance frequency of the leakage flux recovery coil and the capacitor to $250 \mathrm{kHz}$.

Figure 11 shows charging characteristics of the energy harvesting circuit. As a result, the charging voltage $V_{\mathrm{o}}$ of the energy harvesting circuit using COW and MPW was $2.3 \mathrm{~V}$ and $3.9 \mathrm{~V}$, respectively. The electrostatic energy stored in the charging capacitor of the energy harvesting circuit using COW and MPW was $761 \mu \mathrm{J}$ and $265 \mu \mathrm{J}$, respectively. The charging time to the charging capacitor of the energy harvesting circuit using COW and MPW was $40 \mathrm{~s}$ and $130 \mathrm{~s}$, respectively. The reason why the charging time became longer using MPW is that the charging time depends on the drive state of the notebook PC. In the case of using MPW for the windings of the leakage flux recovery coil, the wireless module is activated.

\section{Conclusion}

In this paper, the following were discussed.

1) Impedance characteristics of the leakage flux recov-ery coil

The quality factor $Q$ of the leakage flux recovery coil using COW and MPW at frequency $f=250 \mathrm{kHz}$ was 119 and 135, respectively; thus, the inductance of MPW increased by $13.4 \%$ compared with that of COW. This finding is due to the reduction of the AC resistance and the increase of the inductance of the coil using MPW.
2) Output characteristics of the energy harvesting circuit

The output power of the energy harvesting circuit using COW and MPW at frequency $f=250 \mathrm{kHz}$ and flux density $B=5 \mu \mathrm{T}$ was $679 \mu \mathrm{W}$ and $695 \mu \mathrm{W}$, respectively; thus, the output voltage of MPW increased by $2.3 \%$ compared with that of COW. This finding is due to the increase of the quality factor $Q$ of the coil using MPW.

3) Energy harvesting from a notebook PC

The energy harvesting circuit recovered $3.9 \mathrm{~V}$ charging voltage, as well as the $761 \mu \mathrm{J}$ of electrostatic energy $W$ for the coil using MPW from a notebook PC. In the case of using MPW for the windings of the leakage flux recovery coil, the wireless module is activated.

\section{References}

[1] Y. Suzuki, "Energy Harvesting Handbook," NTS, 2012, pp.3-11, (in Japanese).

[2] K. Tashiro, H. Wakiwaka, S. Inoue, Y. Uchiyama, "Energy Harvesting of Magnetic Power-Line Noise," IEEE Transactions On Magnetics, Vol.47, No.10, pp.4441-4444, 2011.

[3] T. Le, K. Mayaram, T. Fiez, "Efficient Far-Field Radio Frequency Energy Harvesting for Passively Powered Sensor Networks," IEEE Journal of Solid-State Circuits, Vol.46, No.7, pp.1728-1741, 2011.

[4] K. Tashiro, G. Hattori, H. Wakiwaka, "Magnetic flux concentration methods for magnetic energy harvesting module," EPJ Web Conferences, Vol.40, No.06011, 2013.

[5] T. Mizuno, S. Enoki, T. Suzuki, T. Asahina, M. Noda, H. Shinagawa, "Reduction of eddy current loss in magnetoplated wire," Compel-the International Journal for Computation and Mathematics in Electrical and Electronic Engineering, Vol. 28, No. 1, pp.57-66, 2009.

[6] K. Tashiro, A. Matsuoka, H. Wakiwaka, "Simple-Box-9 coil system: A novel approach to design of a square coil system for producing uniform magnetic fields," Materials Science Forum, 670, pp.275-283, 2010. 